\title{
Prognostic Value of Age-Adjusted D-Dimer Cutoff Thresholds in Patients with Acute Coronary Syndrome Treated by Percutaneous Coronary Intervention
}

\author{
Runzhen Chen $\mathbb{D}^{1,2}$, Chen Liu ${ }^{1-3}$, Peng Zhou ${ }^{1,3}$, Jiannan Li', Jinying Zhou', Ruoqi Song ${ }^{4}$, Weida Liu ${ }^{4}$, \\ Yi Chen', Li Song ${ }^{1,3}$, Hanjun Zhao', ${ }^{1,3}$ Hongbing Yan $\mathbb{D}^{2,3}$ \\ 'Department of Cardiology, Fuwai Hospital, National Center for Cardiovascular Diseases, Peking Union Medical College and Chinese Academy of \\ Medical Sciences, Beijing, People's Republic of China; ${ }^{2}$ Fuwai Hospital, Chinese Academy of Medical Sciences, Shenzhen, People's Republic of \\ China; ${ }^{3}$ Coronary Heart Disease Center, Fuwai Hospital, Chinese Academy of Medical Sciences, Beijing, People's Republic of China; ${ }^{4}$ State Key \\ Laboratory of Cardiovascular Disease, Fuwai Hospital, National Center for Cardiovascular Diseases, Peking Union Medical College and Chinese \\ Academy of Medical Sciences, Beijing, People's Republic of China
}

Correspondence: Hanjun Zhao; Hongbing Yan, Email 15210020808@I63.com; hbyanfuwai20I8@I63.com; hbyanfuwai@aliyun.com

Background: Associations between D-dimer and outcomes of patients with acute coronary syndrome (ACS) remain controversial. Using age-adjusted D-dimer cutoff thresholds improve the diagnostic accuracy for thrombotic diseases. This study aimed to investigate the prognostic value of age-adjusted D-dimer in ACS patients treated by percutaneous coronary intervention (PCI).

Methods: A total of 3972 consecutive patients with ACS treated by PCI were retrospectively recruited. The basal age-adjusted D-dimer threshold was $500 \mathrm{ng} / \mathrm{mL}$ and was calculated as age $\times 10$ in patients older than 50 years. Cox regression was used for outcome analysis. C-index, net reclassification index (NRI), and integrated discrimination improvement (IDI) were calculated to assess the additional prognostic value of age-adjusted D-dimer when combined with established clinical risk factors. The primary outcome was all-cause death.

Results: During a median follow-up of 720 days, a total of 225 deaths occurred. High D-dimer level, as defined by age-adjusted thresholds, was an independent predictor for all-cause death (hazard ratio [HR]: 1.75, 95\% confidence interval [CI]: 1.32-2.31, P < 0.001), cardiac death (HR: 1.84, 95\% CI: 1.30-2.60, P =0.001), and MACE (HR: 1.48, 95\% CI: 1.19-1.83, P < 0.001). Sensitivity and subgroup analysis showed that high D-dimer levels were constantly associated with worse outcomes across common risk factors and comorbidities. Besides, age-adjusted elevation of D-dimer significantly improved the risk predictions for all-cause death when added to the model of established risk factors (C-index: 0.846 vs $0.838, \Delta \mathrm{C}$-index: $0.008,95 \% \mathrm{CI}: 0.001-0.015$, $\mathrm{P}_{\text {difference }}=0.027$; NRI: 0.645, 95\% CI: 0.464-0.826, P < 0.001; IDI: 0.008, 95\% CI: $0.001-0.017, \mathrm{P}=0.048$ ).

Conclusion: In ACS patients treated by PCI, age-adjusted elevation of D-dimer was an independent predictor for adverse outcomes and improved the risk predictions for long-term mortality.

Keywords: D-dimer, aging, acute coronary syndrome, percutaneous coronary intervention, PCI, ACS

\section{Introduction}

As the degradation product from cross-linked fibrin, D-dimer has been commonly used for diagnosis and outcome predictions of thrombotic and vascular diseases. ${ }^{1,2}$ Recent studies show that D-dimer level is associated to outcomes of patients with stable coronary heart disease (CHD) treated by percutaneous coronary intervention (PCI), and could improve the risk predictions for long-term adverse events. ${ }^{3,4}$ However, prognostic value of D-dimer remained controversial in the context of acute coronary syndrome (ACS), as previous studies report huge variations regarding cutoff thresholds to define high-risk population, hazards associated with D-dimer elevation, and its efficacy to improve risk predictions. ${ }^{5-13}$ One of the reasons preventing risk stratification based on D-dimer is the confounding due to aging. The 
levels of D-dimer gradually increase along with aging not only in normal population, ${ }^{14,15}$ but also for those with CHD. ${ }^{16}$ In this scenario, higher mortality and risk of ischemia indicated by elevations of D-dimer could be actually due to aging itself, which attenuates the value of D-dimer as a biomarker for clinical diagnosis and risk predictions.

To solve this problem, age-adjusted D-dimer thresholds have been proposed in daily clinical practice. This set of cutoff thresholds is calibrated by adding $10 \mathrm{ng} / \mathrm{mL}$ per 1 year-old increment of age to the base of $500 \mathrm{ng} / \mathrm{mL}$ for patients over 50 years old, which proves to be a convenient and effective approach for the diagnosis and risk stratifications of thrombotic diseases (e.g., pulmonary embolization [PE], deep vein thrombosis [DVT]). ${ }^{1,2,17,18}$ Adjusting D-dimer thresholds by age could be a solution for its application in ACS patients, but relevant evidence is so far limited. To assess the practicability of risk prediction based on age-adjusted D-dimer thresholds, the current study has investigated the followings: (1) relations between age and D-dimer levels after ACS treated by PCI, (2) associations between adverse outcomes and D-dimer elevation defined by age-adjusted thresholds, and (3) whether age-adjusted elevation of D-dimer could improve the risk predictions when combined with established clinical risk factors.

\section{Methods}

\section{Study Cohort}

This retrospective cohort study was based on a cohort in a large-volume PCI center at a national tertiary care institute specializing in cardiovascular diseases (Fuwai Hospital, Beijing, China), which has enrolled all patients undergoing coronary angiography and PCI procedures (emergent or selective). ${ }^{13}$ ACS was diagnosed and classified according to guidelines and universal definitions up to date, including criteria of clinical presentations, typical characteristics on electrocardiography, dynamical changes of cardiac enzymes, and imaging evidence. ${ }^{19-21}$ The ST-segment elevation myocardial infarction (STEMI) was identified by acute chest pain and persistent ( $>20 \mathrm{~min})$ ST-segment elevation, while the non-ST-elevation ACS was identified by acute chest discomfort without persistent ST-segment elevation. All the patients diagnosed with ACS and subsequently undergoing emergent coronary angiography and PCI were included in this study, while those with thrombotic diseases diagnosed during the index hospitalization (i.e., PE, DVT, arterial thrombosis, mural thrombus, and thrombophlebitis) or no follow-up records were excluded. The study was performed in accordance with principles set forth in the Declaration of Helsinki, and was approved by the Ethics Committee of Fuwai Hospital, Peking Union Medical College and Chinese Academy of Medical Sciences (No. 2014-564). All patients had signed the written informed consents during hospitalizations regarding the use of clinical data for the purpose of scientific research by the institute.

\section{Coronary Angiography and PCl Procedure}

Generally, emergent coronary angiography and PCI procedures were performed through radial access. During the procedure, heparin was routinely administered with the dosage of $100 \mathrm{IU} / \mathrm{kg}$. Thrombolysis in Myocardial Infarction (TIMI) grading system was used to assess the antegrade blood flow of the target lesion. The use of certain PCI techniques and relevant devices (e.g., stenting, balloon dilation, thrombus aspiration, intra-aortic balloon pump) were discussed during the procedures and decided by the operators. After the PCI procedures, patients were subsequently transferred to the coronary care unit for further monitoring.

\section{Measurements of D-Dimer}

Blood samples for measurements of D-dimer were routinely collected via the cubital vein as soon as patients were admitted into the coronary care unit after the PCI procedure. Plasma D-dimer level was measured using immunoassay turbidimetry (STA Compact Assay Instrument, Diagnostica Stago Inc, France). Age-adjusted D-dimer threshold was 500 $\mathrm{ng} / \mathrm{mL}$ (fibrinogen-equivalent units) for patients less than 50 years old, and was calculated as age $\times 10 \mathrm{ng} / \mathrm{mL}$ for patients older than 50 years old. High D-dimer level was defined by an elevation of D-dimer surpassing the age-adjusted thresholds. 


\section{Outcomes and Follow-Up}

The primary outcome for this study was all-cause death. The secondary outcomes included cardiac death and major adverse cardiovascular events (MACE), a composite of all-cause death, recurrent myocardial infarction, and ischemic stroke. The patient would be routinely followed up at 1,6, and 12 months after discharge, either by outpatient visits or phone call interview. Follow-up was also performed during rehospitalizations and outpatient visits at the institute due to adverse events or re-examinations. For those who survived more than a year, the subsequent follow-up would be made annually. A group of three physicians routinely assessed the reported adverse events.

\section{Statistical Analysis}

Restrictive cubic spline (RCS) was used to detect the possible nonlinear association between age and D-dimer levels after ACS. Univariable Cox regression was used to assess the association between levels of D-dimer and clinical outcomes, followed by multiple adjustments for established risk factors of adverse events after ACS and PCI, ${ }^{19,21}$ including age, sex, diabetes, hypertension, peripheral artery diseases, diagnosis of STEMI, atrial fibrillation, old myocardial infarction, history of stroke, incidence of cardiac arrest, ejection fraction (EF), creatinine, peak value of cardiac troponin I, lowdensity lipoprotein cholesterol, high-sensitivity C-reactive protein (hsCRP), culprit lesion, multivessel disease, pre- and post-procedure TIMI flow, and door-to-balloon time. To evaluate the additional prognostic value of age-adjusted elevation of D-dimer when incorporated to models established by clinical risk factors, the C-index, net reclassification index (NRI), and integrated discrimination improvement (IDI) were calculated for the primary outcome. C-index was calculated and compared as described by Newson, ${ }^{22}$ where Somer's D of the model was firstly generated and then transformed into Harrell's C. As there are no pre-defined clinical risk thresholds for models including age-adjusted D-dimer, continuous NRI and IDI was calculated at the median follow-up. NRI and IDI calculation was performed with a Stata add-on based on the methods developed by Pencina et al. ${ }^{23,24}$ Details of sample size calculations are shown in Supplementary Figure 1. Categorical variables are presented as numbers (\%) and analyzed with chi-square test. Continuous variables are presented using mean \pm SD if they follow the normal distribution and tested with Student's $t$-test. Otherwise, they are presented as medians with $25^{\text {th }}$ and $75^{\text {th }}$ percentiles and tested by Wilcoxon rank sum tests. Multiple imputations were performed for missing values of lab test results using the mi command in Stata. All the statistical analyses were performed using Stata (version 15.0, StataCorp, College Station, TX, USA). A P-value $<0.05$ was considered statistically significant.

\section{Results}

\section{Patient Cohort and Baseline Characteristics}

From January 2010 to June 2017, a total of 4151 patients had been admitted to the institute to receive emergent coronary angiography and PCI due to ACS. Among these patients, 86 (2.1\%) patients were diagnosed with thrombotic diseases during the index hospitalization, and 93 (2.2\%) patients did not have follow-up records in any forms (i.e., phone-call interview, outpatient visits, or re-hospitalizations at the institute). Finally, a total of 3972 (95.7\%) patients were included in the current analysis. The baseline characteristics are shown in Table 1. The patient mean age was $59.0 \pm 11.9$ years, and $78.9 \%$ of the patients were male. Overall, the level of post-procedural D-dimer was $330(220-590) \mathrm{ng} / \mathrm{mL}$. Regression using RCS methods depicted a nonlinear association $(\mathrm{P}$ nonlinearity $=0.009)$ between D-dimer and age (Figure 1). The post-PCI D-dimer level after ACS was similar in younger ages, but began to increase along with aging at around 55 to 60 years old. According to the age-adjusted thresholds, 914 (23.0\%) patients were defined to have high D-dimer levels. According to the sample size curve (Supplementary Figure 1), the current sample size was adequate to detect a hazard increase of $50 \%$ to two folds at a power of $80 \%$ for a two-sided Log rank test with $\alpha=0.05$. Patients with high D-dimer tended to be older, female, more often complicated with hypertension, atrial fibrillation, peripheral artery disease, previous strokes, tumors, and worse hemodynamics, along with higher levels of creatinine and hsCRP. Right coronary artery (41.1\%) and left anterior descending artery $(42.6 \%)$ constituted the major culprit lesions for patients with D-dimer elevation, while both the pre- and post-procedure antegrade coronary blood flow was worse if D-dimer was elevated. Moreover, patients with high D-dimer received more invasive treatments of intra-aortic balloon 
Table I Baseline Characteristics of Study Patients According to D-Dimer Levels Defined by Age-Adjusted Thresholds

\begin{tabular}{|c|c|c|c|c|}
\hline Variables & Overall (N=3972) & $\begin{array}{l}\text { Low D-Dimer } \\
\qquad(\mathbf{N}=\mathbf{3 0 5 8 )}\end{array}$ & $\begin{array}{l}\text { High D-Dimer } \\
\qquad(\mathrm{N}=9 \mid 4)\end{array}$ & P-value \\
\hline Age, years & $59.0 \pm 11.9$ & $58.3 \pm 11.8$ & $61.4 \pm 12.2$ & $<0.001$ \\
\hline Male sex, n (\%) & $3132(78.9)$ & $2485(81.3)$ & $647(70.8)$ & $<0.001$ \\
\hline Diabetes mellitus, n (\%) & $1299(32.7)$ & $989(32.3)$ & $310(33.9)$ & $<0.37$ \\
\hline Hypertension, n (\%) & $2429(61.2)$ & $1835(60.0)$ & $594(65.0)$ & 0.007 \\
\hline Atrial fibrillation, $\mathrm{n}(\%)$ & $227(5.7)$ & I $38(4.5)$ & $89(9.7)$ & $<0.001$ \\
\hline Peripheral artery diseases, $\mathrm{n}(\%)$ & $159(4.0)$ & $102(3.3)$ & $57(6.2)$ & $<0.001$ \\
\hline Old myocardial infarction, n (\%) & $537(13.5)$ & $400(13.1)$ & $137(15.0)$ & 0.14 \\
\hline History of stroke, n (\%) & $531(13.4)$ & $363(11.9)$ & $168(18.4)$ & $<0.001$ \\
\hline History of CABG or $\mathrm{PCl}, \mathrm{n}(\%)$ & $583(14.7)$ & $449(14.7)$ & $134(14.7)$ & 0.99 \\
\hline STEMI, n (\%) & $3467(87.3)$ & $2649(86.6)$ & $818(89.5)$ & 0.022 \\
\hline Tumor diseases, $\mathrm{n}(\%)$ & $81(2.0)$ & $54(1.8)$ & $27(3.0)$ & 0.026 \\
\hline Liver diseases, n (\%) & $570(14.4)$ & $453(14.8)$ & $117(12.8)$ & 0.13 \\
\hline COPD, n (\%) & $32(0.8)$ & $24(0.8)$ & $8(0.9)$ & 0.79 \\
\hline \multicolumn{5}{|l|}{ Hemodynamics } \\
\hline Heart rate, bpm & $77.4 \pm 15.2$ & $76.1 \pm 14.2$ & $81.6 \pm 17.7$ & $<0.001$ \\
\hline Systolic blood pressure, $\mathrm{mmHg}$ & $125.7 \pm 18.3$ & $125.0 \pm 18.1$ & $123.7 \pm 19.2$ & 0.060 \\
\hline $\mathrm{EF}, \%$ & $53.8 \pm 7.6$ & $54.5 \pm 7.1$ & $51.5 \pm 8.7$ & $<0.001$ \\
\hline Cardiac arrest, n (\%) & $144(3.6)$ & $81(2.6)$ & $63(6.9)$ & $<0.001$ \\
\hline \multicolumn{5}{|l|}{ Laboratory tests } \\
\hline D-dimer, $n g / m L$ & $330(220-590)$ & $270(200-380)$ & $1050(790-1800)$ & $<0.001$ \\
\hline LDL-C, $\mathrm{mmol} / \mathrm{L}$ & $2.7 \pm 0.9$ & $2.7 \pm 0.9$ & $2.8 \pm 1.0$ & 0.27 \\
\hline hsCRP, mg/L & $7.16(2.75-12.03)$ & $5.24(2.50-11.70)$ & $10.73(4.00-12.86)$ & $<0.001$ \\
\hline Creatinine, $\mu \mathrm{mol} / \mathrm{L}$ & $82.0 \pm 25.1$ & $80.5 \pm 21.3$ & $86.8 \pm 34.5$ & $<0.001$ \\
\hline Peak cTnl, ng/mL & $2.505(0.44 \mid-10.803)$ & $2.370(0.424-9.850)$ & $3.390(0.509-17.500)$ & $<0.001$ \\
\hline \multicolumn{5}{|l|}{ Coronary angiographic findings } \\
\hline \multicolumn{5}{|l|}{ Culprit lesion, n (\%) } \\
\hline Left main artery & $95(2.4)$ & $63(2.1)$ & $32(3.5)$ & 0.002 \\
\hline Left anterior descending artery & $1734(43.7)$ & $1345(44.0)$ & $389(42.6)$ & \\
\hline Left circumflex & $610(15.4)$ & $498(16.3)$ & $112(12.3)$ & \\
\hline Right coronary artery & $1515(38.1)$ & $1139(37.2)$ & $376(4 I . I)$ & \\
\hline Bypass graft & $18(0.5)$ & $13(0.4)$ & $5(0.5)$ & \\
\hline \multicolumn{5}{|l|}{ Multi-vessel disease, n (\%) } \\
\hline I-vessel disease & $1002(25.2)$ & $784(25.6)$ & $218(23.9)$ & 0.51 \\
\hline 2-vessel disease & $1253(31.5)$ & $964(31.5)$ & $289(31.6)$ & \\
\hline 3-vessel disease & $1717(43.2)$ & $1310(42.8)$ & $407(44.5)$ & \\
\hline Pre-PCI TIMI 0 flow, n (\%) & $2607(65.6)$ & $1979(64.7)$ & $628(68.7)$ & 0.026 \\
\hline Post-PCI TIMI 3 flow, n (\%) & $3822(96.2)$ & $2953(96.6)$ & $869(95.1)$ & 0.038 \\
\hline Door-to-balloon time, mins & $128(95-202)$ & $130(95-208)$ & $124(90-189)$ & 0.005 \\
\hline Stent placement, n (\%) & $3497(88.0)$ & $2726(89.1)$ & $771(84.4)$ & $<0.001$ \\
\hline Thrombus aspiration, $\mathrm{n}(\%)$ & $1649(41.5)$ & $1240(40.5)$ & 409 (44.7) & 0.024 \\
\hline IABP, n (\%) & $381(9.6)$ & $205(6.7)$ & $176(19.3)$ & $<0.001$ \\
\hline Glycoprotein Ilb/Illa inhibitors, n (\%) & $534(13.4)$ & $408(13.3)$ & $126(13.8)$ & 0.73 \\
\hline $\begin{array}{l}\text { Complete revascularization before discharge, } \\
\mathrm{n}(\%)\end{array}$ & $|73|(43.6)$ & $1369(44.8)$ & $362(39.6)$ & 0.006 \\
\hline
\end{tabular}


Table I (Continued).

\begin{tabular}{|c|c|c|c|c|}
\hline Variables & Overall $(\mathrm{N}=3972)$ & $\begin{array}{c}\text { Low D-Dimer } \\
(\mathrm{N}=\mathbf{3 0 5 8 )}\end{array}$ & $\begin{array}{l}\text { High D-Dimer } \\
\quad(N=9 \mid 4)\end{array}$ & P-value \\
\hline \multicolumn{5}{|l|}{ Medications } \\
\hline Aspirin, n (\%) & 3930 (98.9) & 3034 (99.2) & $896(98.0)$ & 0.002 \\
\hline P2YI2 inhibitors, n (\%) & $3936(99.1)$ & $3037(99.3)$ & $899(98.4)$ & 0.008 \\
\hline Statins, n (\%) & $3708(93.4)$ & $2869(93.8)$ & $839(91.8)$ & 0.031 \\
\hline ACEI/ARB, $n(\%)$ & $2813(63.4)$ & $2234(73.1)$ & $579(63.4)$ & $<0.001$ \\
\hline$\beta$-blockers, n (\%) & $3468(87.3)$ & $2699(88.3)$ & $769(84.1)$ & 0.001 \\
\hline Anticoagulants, n (\%) & $19(0.5)$ & $17(0.6)$ & $2(0.2)$ & 0.195 \\
\hline
\end{tabular}

Note: Medications typically referred to drugs prescribed at discharge, or otherwise, the drugs being used during hospitalization if patients died before discharge.

Abbreviations: ACEI/ARB, angiotensin-converting enzyme inhibitors and angiotensin receptor blockers, bpm, beats per minute; CABG, coronary artery bypass grafting; COPD, chronic obstructive pulmonary disease; cTnl, cardiac troponin I; EF, ejection fraction; hsCRP, high sensitivity C-reactive protein; IABP, intra-aortic balloon pump; LDLC, low-density lipoprotein cholesterol; PCI, percutaneous coronary intervention; STEMI, ST-segment elevation myocardial infarction; TIMI flow, Thrombolysis In Myocardial Infarction grade flow.

pump and thrombus aspiration, but less interventions of stent placements and complete revascularization. Regarding medications, the use of aspirin, P2Y12 inhibitors, statins and $\beta$-blockers were slightly less frequent in patients with high D-dimer, while the use of renin-angiotensin blockade medications was more prevalent in patients with lower D-dimer, and the use of anticoagulants was similar between the two groups.

\section{Associations Between Age-Adjusted Elevation of D-Dimer and Clinical Outcomes}

During a median follow-up of 720 days, there were 225 deaths (5.7\%, incidence rate [IR]: 17.60/1000-person-years [PY]), 151 cardiac deaths (3.8\%, IR: 11.81/1000 PY), and 408 MACEs (10.3\%, IR: 31.91/1000 PY) in total. Associations between D-dimer and various outcomes are shown in Table 2. Univariable analysis showed that high D-dimer level, as

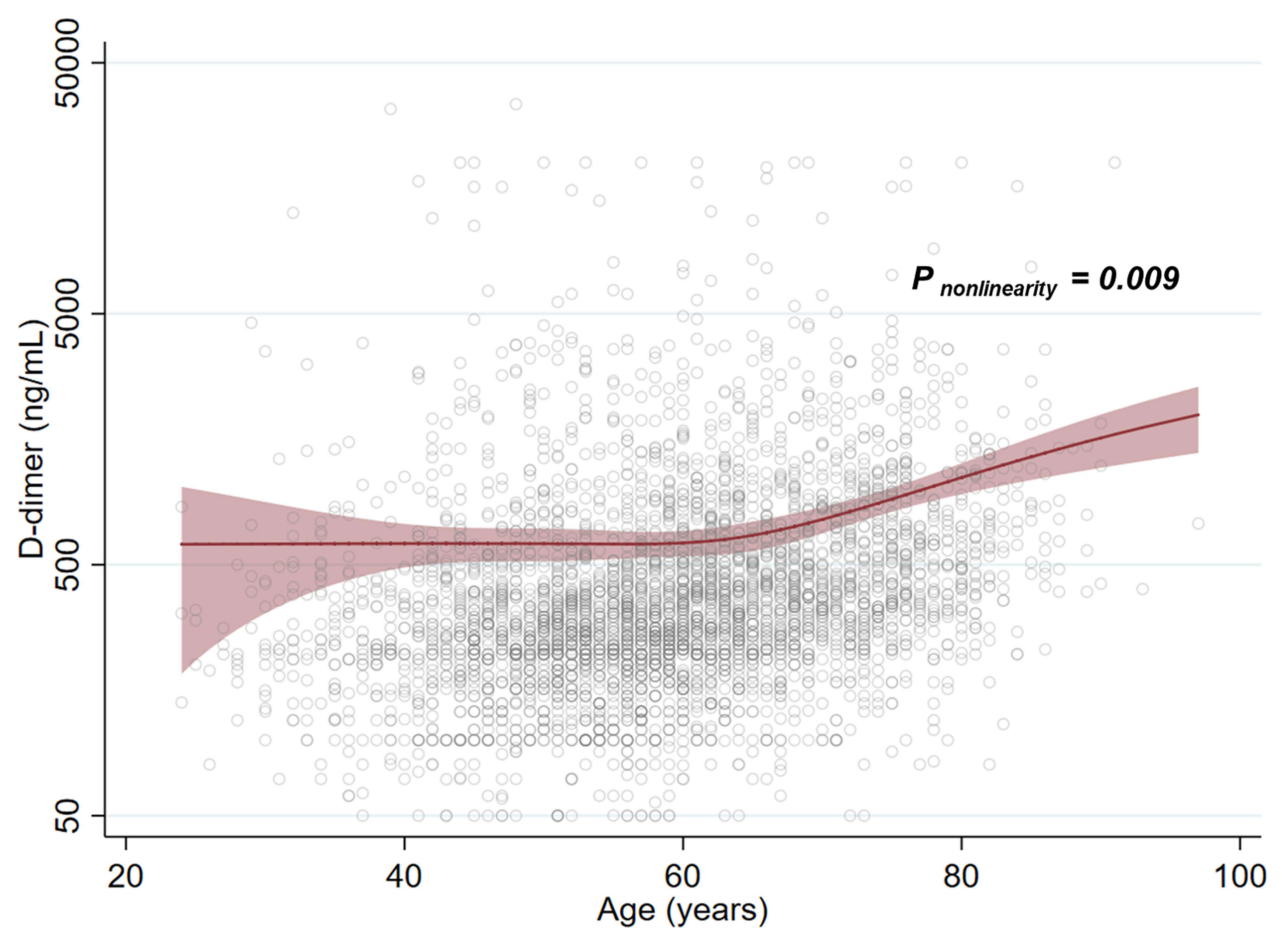

Figure I Relationship between post-procedural D-dimer and age in patients with acute coronary syndrome treated by percutaneous coronary intervention. Circle $(O)=$ actual D-dimer level, line $=\mathrm{D}$-dimer levels predicted by restrictive cubic spline regression, dashed area $=95 \%$ confidence interval. 
Table 2 Clinical Outcomes According to D-Dimer Levels Defined by Age-Adjusted Thresholds

\begin{tabular}{|c|c|c|c|c|c|c|c|}
\hline $\begin{array}{l}\text { Level of } \\
\text { D-Dimer }\end{array}$ & $\begin{array}{c}\text { Incidence Rate } \\
\text { (/1000 PY) }\end{array}$ & $\begin{array}{c}\text { Unadjusted HR } \\
(95 \% \mathrm{CI})\end{array}$ & P-value & $\begin{array}{c}\text { Model I HR } \\
(95 \% \mathrm{CI})\end{array}$ & P-value & $\begin{array}{c}\text { Model } 2 \text { HR } \\
(95 \% \mathrm{Cl})\end{array}$ & P-value \\
\hline \multicolumn{8}{|l|}{ All-cause death } \\
\hline Low D-dimer & 12.24 & I (reference) & - & I (reference) & - & I (reference) & - \\
\hline High D-dimer & 37.24 & $2.99(2.30-3.88)$ & $<0.001$ & $1.75(|.32-2.3|)$ & $<0.001$ & $1.61(1.20-2.15)$ & 0.001 \\
\hline Log (D-dimer) & - & $1.68(1.52-1.86)$ & $<0.001$ & $1.28(1.12-1.46)$ & $<0.001$ & $1.22(1.06-1.39)$ & 0.004 \\
\hline \multicolumn{8}{|l|}{ Cardiac death } \\
\hline Low D-dimer & 7.57 & I (reference) & - & I (reference) & - & I (reference) & - \\
\hline High D-dimer & 27.38 & $3.5 \mathrm{I}(2.55-4.83)$ & $<0.001$ & $1.84(1.30-2.60)$ & 0.001 & $1.67(1.16-2.39)$ & 0.006 \\
\hline Log (D-dimer) & - & $1.77(1.57-2.00)$ & $<0.001$ & $1.30(1.10-1.53)$ & 0.002 & $1.22(1.03-1.45)$ & 0.019 \\
\hline \multicolumn{8}{|l|}{ MACE } \\
\hline Low D-dimer & 26.38 & I (reference) & - & I (reference) & - & I (reference) & - \\
\hline High D-dimer & 52.21 & $1.98(1.61-2.43)$ & $<0.001$ & $1.48(1.19-1.83)$ & $<0.001$ & $1.38(1.11-1.71)$ & 0.004 \\
\hline Log (D-dimer) & - & $1.40(1.29-1.52)$ & $<0.001$ & $1.15(1.04-1.27)$ & 0.005 & $1.12(1.01-1.24)$ & 0.030 \\
\hline
\end{tabular}

Notes: Model I, adjusted for established risk factors, including age, sex, diabetes, hypertension, peripheral artery diseases, diagnosis of ST-segment elevation myocardial infarction, atrial fibrillation, old myocardial infarction, history of stroke, incidence of cardiac arrest, ejection fraction, creatinine, peak value of cardiac troponin I, low-density lipoprotein cholesterol, high-sensitivity C-reactive protein, culprit lesion, multivessel disease, pre- and post-procedure TIMI flow, door-to-balloon time; Model 2, adjusted for all baseline variables.

Abbreviations: HR, hazard ratio; MACE, major adverse cardiovascular events; PY, person years; TIMI grade flow, Thrombolysis In Myocardial Infarction grade flow.

defined by age-adjusted thresholds, was associated with substantially higher mortality and incidence of MACE. The Kaplan-Meier survival analysis showed a significantly higher incidence of primary and secondary endpoint events (all $\mathrm{P}_{\text {log-rank }}<0.001$ ) in the group with high D-dimer levels (Figure 2). Associations between various outcomes and D-dimer levels sustained after adjustments for established risk factors. Age-adjusted elevation of D-dimer was an independent predictor for increased risk of all-cause death (HR:1.75, 95\% CI:1.32-2.31, P $<0.001$ ), cardiac death (HR: 1.84, 95\% CI: 1.30-2.60, $\mathrm{P}=0.001$ ), and MACE (HR: $1.48,95 \% \mathrm{CI}: 1.19-1.83, \mathrm{P}<0.001$ ). When treated as a continuous variable, logarithmic D-dimer was still independently associated with higher incidence of adverse events. These associations between D-dimer and various outcomes remained robust in models fully adjusted for all collected baseline variables. Sensitivity analysis further showed that age-adjusted elevation of D-dimer was still associated with higher incidence of adverse outcomes when patients with certain comorbidities and adverse events during the acute phase of the index ACS were excluded (Supplementary Table 1).

According to the subgroup analysis for the primary outcome (Figure 3), the increase of hazards associated with ageadjusted elevation of D-dimer was generally consistent across demographics and common comorbidities (e.g., age,
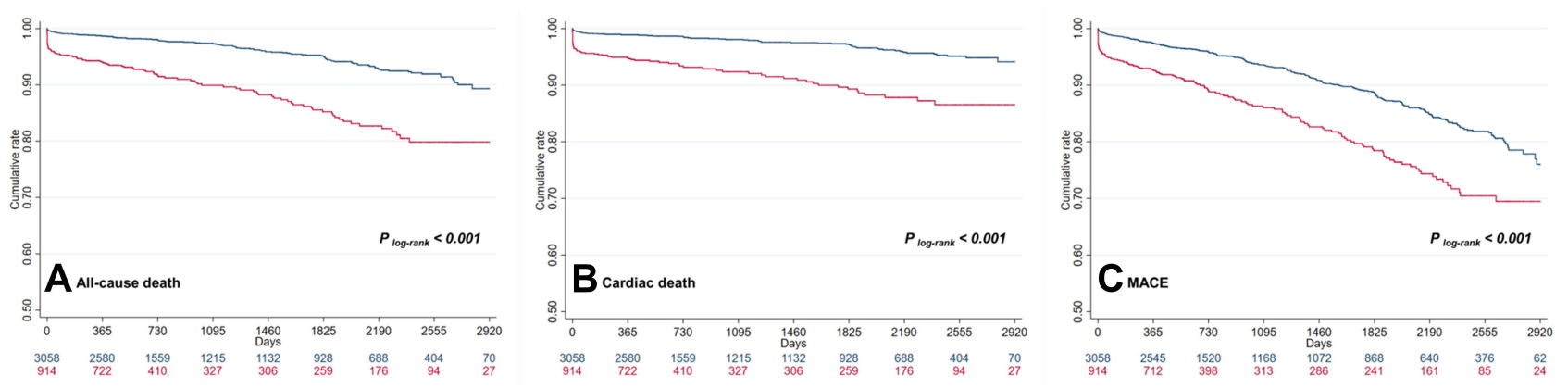

Figure 2 Kaplan-Meier survival curves of primary and secondary outcomes according to D-dimer levels. (A) Survival curves for the outcome of all-cause death. (B) Survical curves for the outcome of cardiac death. (C) Survival curves for the outcome of MACE.

Abbreviation: MACE, major adverse cardiovascular events. 
A All-cause death

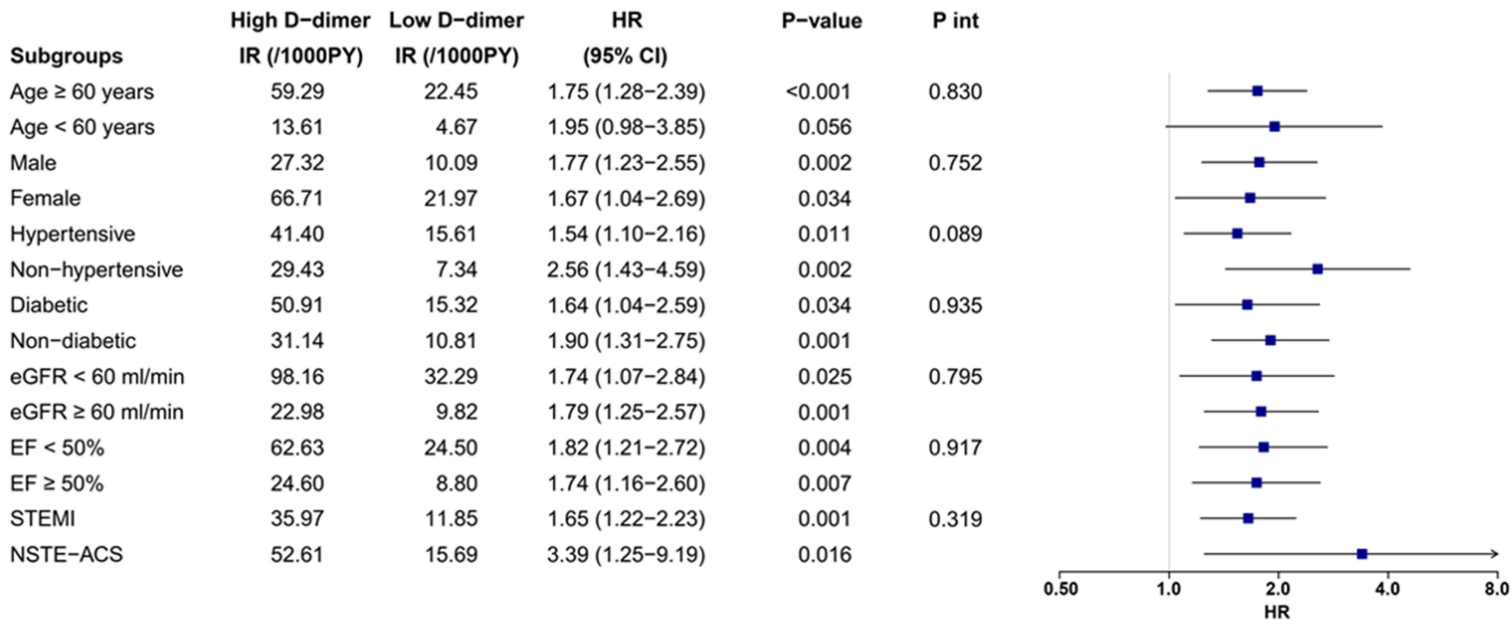

\section{B Cardiac death}

$\begin{array}{lccccc}\text { Subgroups } & \begin{array}{c}\text { High D-dimer } \\ \text { IR (/1000PY) }\end{array} & \begin{array}{c}\text { Low D-dimer } \\ \text { IR (/1000PY) }\end{array} & \begin{array}{c}\text { HR } \\ \mathbf{( 9 5 \% ~ C l ) ~}\end{array} & \text { P-value } & \text { P int } \\ \text { Age } \geq 60 \text { years } & 43.77 & 13.80 & 1.92(1.31-2.83) & 0.001 & 0.806 \\ \text { Age }<60 \text { years } & 9.83 & 2.95 & 1.49(0.63-3.53) & 0.359 & \\ \text { Male } & 18.05 & 6.32 & 1.63(1.02-2.58) & 0.040 & 0.296 \\ \text { Female } & 55.11 & 13.18 & 2.22(1.25-3.94) & 0.007 & \\ \text { Hypertensive } & 31.89 & 9.40 & 1.86(1.24-2.79) & 0.003 & 0.478 \\ \text { Non-hypertensive } & 18.92 & 4.89 & 2.22(1.02-4.80) & 0.044 & \\ \text { Diabetic } & 40.25 & 7.82 & 2.21(1.24-3.94) & 0.007 & 0.213 \\ \text { Non-diabetic } & 21.64 & 7.45 & 1.74(1.11-2.74) & 0.016 & \\ \text { eGFR }<60 \mathrm{ml} / \mathrm{min} & 78.91 & 18.45 & 2.29(1.26-4.16) & 0.007 & 0.291 \\ \text { eGFR } \geq 60 \mathrm{ml} / \mathrm{min} & 15.32 & 6.25 & 1.66(1.05-2.61) & 0.029 & \\ \text { EF }<50 \% & 48.35 & 17.69 & 1.87(1.17-2.99) & 0.009 & 0.530 \\ \text { EF } \geq 50 \% & 16.95 & 4.72 & 2.23(1.33-3.74) & 0.002 & \\ \text { STEMI } & 26.48 & 7.09 & 1.75(1.20-2.55) & 0.004 & 0.638 \\ \text { NSTE-ACS } & 38.26 & 11.77 & 2.94(0.91-9.56) & 0.072 & \end{array}$

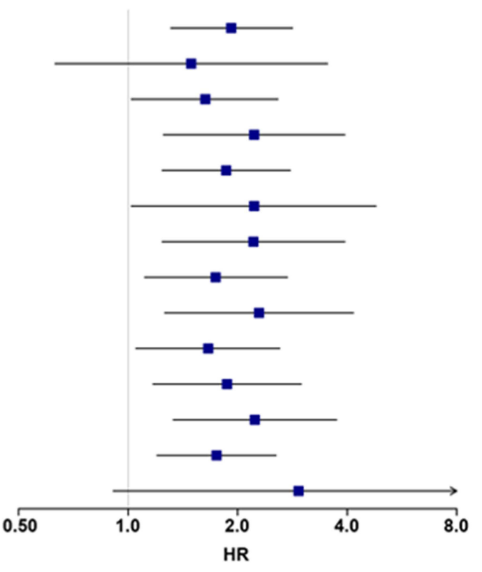

\section{MACE}

$\begin{array}{lcccc}\text { Subgroups } & \begin{array}{c}\text { High D-dimer } \\ \text { IR (/1000PY) }\end{array} & \begin{array}{c}\text { Low D-dimer } \\ \text { IR }\end{array} & \begin{array}{c}\text { HR } \\ (\mathbf{9 0 0 0} \% \mathbf{P I})\end{array} & \text { P-value } \\ \text { Age } \geq 60 \text { years } & 76.95 & 38.59 & 1.56(1.21-2.01) & 0.001 \\ \text { Age <60 years } & 25.71 & 17.33 & 1.30(0.85-1.98) & 0.226 \\ \text { Male } & 40.01 & 24.56 & 1.30(1.00-1.71) & 0.053 \\ \text { Female } & 88.47 & 34.60 & 1.64(1.12-2.42) & 0.012 \\ \text { Hypertensive } & 56.50 & 30.55 & 1.39(1.07-1.80) & 0.013 \\ \text { Non-hypertensive } & 44.14 & 20.30 & 1.75(1.17-2.63) & 0.007 \\ \text { Diabetic } & 69.85 & 31.58 & 1.50(1.05-2.13) & 0.024 \\ \text { Non-diabetic } & 44.34 & 23.95 & 1.46(1.11-1.93) & 0.007 \\ \text { eGFR < 60 mI/min } & 115.48 & 43.37 & 1.73(1.13-2.67) & 0.012 \\ \text { eGFR } \geq 60 \mathrm{mi} / \mathrm{min} & 37.40 & 24.32 & 1.34(1.03-1.74) & 0.030 \\ \text { EF < 50\% } & 68.13 & 40.83 & 1.32(0.93-1.87) & 0.122 \\ \text { EF } \geq 50 \% & 44.29 & 22.32 & 1.60(1.21-2.11) & 0.001 \\ \text { STEMI } & 50.20 & 25.37 & 1.43(1.14-1.80) & 0.002 \\ \text { NSTE-ACS } & 76.53 & 35.30 & 1.97(1.03-3.77) & 0.042\end{array}$

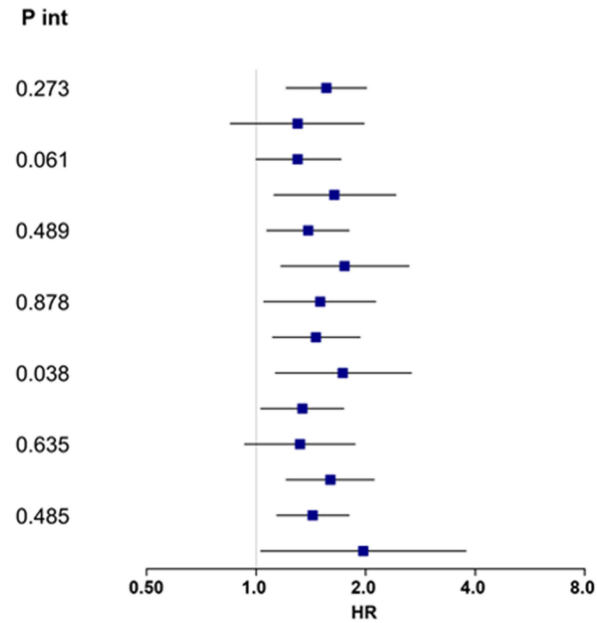

Figure 3 Subgroup analysis for outcomes and D-dimer levels across common clinical risk factors. (A) Associations between age-adjusted elevation of D-dimer and all-cause death. (B) Associations between age-adjusted elevation of D-dimer and cardiac death. (C) Associations between age-adjusted elevation of D-dimer and MACE. HR was adjusted for established risk factors.

Abbreviations: $\mathrm{Cl}$, confidence interval; EF, ejection fraction; eGFR, estimated glomerular filtration rate; HR, hazard ratio; IR, incidence rate; MACE, major adverse cardiovascular events; PY, person years; P int, P for interactions; STEMI, ST-segment elevation myocardial infarction; NSTE-ACS, non-ST-elevation acute coronary syndrome. 
gender, hypertension, diabetes, estimated glomerular filtration rate [eGFR] $<60 \mathrm{~mL} /\left[\mathrm{min} \cdot 1.73 \mathrm{~m}^{2}\right], \mathrm{EF}<50 \%$, types of

ACS). No significant interactions were observed between D-dimer levels and various subgroups (all $\mathrm{P}$ interaction $>0.05$ ). Similar results were observed for secondary outcomes, except significant interactions in terms of eGFR $(\mathrm{P}$ interaction $=$ 0.038) for the endpoint of MACE, where patients with eGFR $<60 \mathrm{~mL} / \mathrm{min}$ showed higher risk of MACE (HR: 1.73, 95\% CI: $1.13-2.67, \mathrm{P}=0.012)$, as compared to those with higher eGFR $(\geq 60 \mathrm{~mL} / \mathrm{min}, \mathrm{HR}: 1.34,95 \% \mathrm{CI}: 1.03-1.74, \mathrm{P}=$ $0.030)$.

\section{Additional Prognostic Value of Age-Adjusted Elevation of D-Dimer}

Prognostic accuracy for the primary outcome of all-cause death was assessed when age-adjusted elevation of D-dimer was added to the model of established risk factors. The new model incorporating age-adjusted elevation of D-dimer showed favorable performance (C-index: 0.846, 95\% CI: 0.820-0.872, P < 0.001) for the prediction of all-cause death, which was significantly better than that of the original model with established risk factors $(\mathrm{C}$-index: $0.838,95 \% \mathrm{CI}$ : $0.811-0.865$, P < 0.001; $\Delta$ C-index: 0.008, 95\% CI: 0.001-0.015, $\mathrm{P}$ difference $=0.027)$. The NRI (0.645, 95\% CI: $0.464-$ $0.826, \mathrm{P}<0.001)$ and IDI $(0.008,95 \% \mathrm{CI}: 0.001-0.017, \mathrm{P}=0.048)$ also suggested that age-adjusted elevation of $\mathrm{D}-\mathrm{dimer}$ improved the risk predictions for long-term mortality.

\section{Discussions}

The major findings of this study are as follow. For ACS patients treated by PCI: (1) post-procedural D-dimer level was nonlinearly associated with age, (2) the age-adjusted elevation of D-dimer, as defined by D-dimer levels higher than the age-adjusted thresholds, was an independent predictor for mortality and MACE, and (3) age-adjusted D-dimer levels (high vs. low) provided additional prognostic value when combined with established clinical risk factors.

\section{Associations Between D-Dimer Levels and Age}

Due to the changes in microcirculation and blood coagulation, the normal ranges of D-dimer level gradually increase with aging in general population, which is also seen in patients with CHD. ${ }^{14-16}$ Recently, we have also demonstrated that higher D-dimer levels were associated with worse outcomes in ACS-PCI patients, which could also improve the long-term risk predictions. ${ }^{12,13}$ In this study, we furtherly showed that D-dimer levels after ACS were positively correlated with age in a nonlinear pattern. According to RCS analysis, post-PCI D-dimer levels did not variate a lot in younger ages, but start to increase over the age of 55 to 60 . Hence, using different cutoff values according to patient age could be a more reasonable way for applying D-dimer to risk stratifications in ACS patients, as it could help to exclude low-risk elderly patients with apparent elevation of D-dimer. Notably, the median level of D-dimer was 330 (220-590) $\mathrm{ng} / \mathrm{mL}$, which was well below the threshold of $500 \mathrm{ng} / \mathrm{mL}$ to diagnose PE or DVT, but quite close to that of stable CHD patients. ${ }^{3,25}$ These findings suggested that the intra-coronary thrombus might have posed limited impacts on systemic levels of D-dimer, and the elevation of D-dimer was more of a reflection for intrinsic prothrombotic tendency.

\section{D-Dimer, Mortality, and Incidence of Cardiovascular Events}

For a long time, D-dimer is considered too unspecific to use in clinical management of CHD. ${ }^{26,27}$ Although many studies have shown that elevated D-dimer is associated to adverse events in CHD or ACS patients, conflicting results have been reported due to high heterogeneity among studies, regarding clinical presentations of patients (e.g., chest pain, ACS, STsegment elevation ACS, etc.), treating strategies (e.g., PCI, coronary bypass grafting, noninvasive approaches), and outcomes for analysis (e.g., no-reflow, death, in-hospital or long-term MACE). ${ }^{3-11,13}$ In studies supporting a positive role of D-dimer, the thresholds to define an elevation vary from 436 to $1150 \mathrm{ng} / \mathrm{mL}$, with huge variations in relative increase of risk (1.83 to 10.1 folds) compared with the "reference group". ${ }^{5-8,10,11,13}$ In this study, however, only ACS patients treated by emergent PCI had been recruited, constituting a typical patient cohort following the mainstream and standardized clinical practice. The relative risk increased for 75\%, 84\% and 48\% in all-cause death, cardiac death, and MACE, respectively, if the post-procedural D-dimer levels were higher than the age-adjusted thresholds, which was generally equivalent to the risk increment of middle terciles or quartiles of D-dimer in previous studies. ${ }^{3,13,28}$ In sum, our 
results were generally in line with previous reports that D-dimer elevations could predict long-term adverse events after ACS, while the highly homogenous patient profiles and large sample size increased the reliability of these findings.

As previous studies have been focusing on demonstrating the prognostic value of D-dimer, various percentiles have been arbitrarily selected as the cut points to stratify patients into subgroups, which does not allow a unified definition of high-risk population. In this study, D-dimer thresholds were allowed more flexibility due to the calibration of aging itself, as well as potential impacts coming from relevant comorbidities commonly seen in the elderlies. Accordingly, the current study reported a consistent increase of hazard for adverse events despite the impact of aging. In the subgroup analysis, age-adjusted elevation of D-dimer was associated with $75 \%$ increase in risk of death for older ( $\geq 60$ years old) patients, and $95 \%$ increase for younger $(<60$ years old) patients, while no interactions were detected $(\mathrm{P}$ interaction $=0.830)$. Normally, patients with higher D-dimer tend to be older and complicated with more comorbidities, which naturally and inevitably associates to higher mortality ${ }^{14,15}$ while younger patients with elevated risk of thrombosis might be neglected, as they are automatically classified as low-risk population due to the naturally lower levels of D-dimer in younger age. ${ }^{15,16}$ Treatment selections and clinical decision making are therefore difficult based on measurements of D-dimer, as physicians could hardly tell whether the increased risk of ischemia is simply due to aging, or the truly more active thrombosis that needs to be tackled by more intensive antithrombotic medications. However, the current study showed a homogenous increase of hazard related to age-adjusted D-dimer elevation despite aging, suggesting that age-adjusted D-dimer threshold was an effective approach for excluding impacts of aging on D-dimer levels and risk of ischemia, which could potentially reduce overtreatment in older patients and identify younger high-risk patients in need of extra attention.

Notably, significant interactions were observed between D-dimer elevation and eGFR ( $\geq 60 \mathrm{~mL} / \mathrm{min}$ vs $<60 \mathrm{~mL} / \mathrm{min}$ ) for the endpoint of MACE. Patients with eGFR $<60 \mathrm{~mL} / \mathrm{min}$ acquired a $73 \%$ increase in risk of MACE if D-dimer was elevated, while those with comparatively better renal function acquired a risk increase of $34 \%$. Previously, Naruse et al have reported that patients with chronic kidney disease complicated with D-dimer elevation could acquire a one-to-threefold increase in risk of death, which is substantially higher than those with D-dimer elevation alone. ${ }^{3}$ Meanwhile, some studies also show that prolonged use of P2Y12 inhibitors further lower the risk of death and stent thrombosis, while others suggest extended dual antiplatelet treatment (DAPT) does not bring extra clinical benefit but increase risk of bleedings. ${ }^{29-31}$ These findings suggest there is still heterogeneity within the high-risk patients of renal impairment. On top of kidney disease, age-adjusted elevation of D-dimer might serve as an indicator to identify patients at higher risk who are potentially suitable for prolonged DAPT or even anticoagulation, while standard 12-month DAPT or deescalated monotherapy could be considered for those without hypercoagulative status. Taken together, patients with age-adjusted elevation of D-dimer might require extra attention and more intensive care when complicated with impaired renal function.

\section{Improvement for Risk Predictions Using Age-Adjusted D-Dimer Thresholds}

Previous studies show that D-dimer could improve risk predictions for adverse events in patients with stable CHD., Recent studies in ACS patients reveal that D-dimer could improve outcome predictions when combined with clinical risk score tools predicting in-hospital and long-term mortality. ${ }^{13,32}$ In this research, we furtherly demonstrated that ageadjusted elevation of D-dimer significantly improved the predictions for all-cause death when added to models of established clinical risk factors, as shown by the significant increase of C-index. Further tests using NRI and IDI also supported a positive role of age-adjusted elevation of D-dimer for improving outcome predictions. Although the increase of C-index brought by the incorporation of age-adjusted elevation of $\mathrm{D}$-dimer was relatively small ( $\triangle \mathrm{C}$-index $=0.008)$, it should be noticed that the original model already showed favorable performance (C-index: 0.838), which could lead to insensitivity for detecting improvements by adding new biomarkers or predictors. ${ }^{33,34}$

Reasons for these improvements could be multiple, but they boiled down to the extra prognostic information provided by D-dimer. The interaction between coagulation system and blood vessels is one of the major interpretations. Pathological studies show D-dimer and fibrin degradation products are associated to plaque instability. Kothari et al show in an intravascular ultrasound study that D-dimer levels are positively associated with the presence of necrotic core and calcification in coronary plaques. ${ }^{35}$ Corban et al also observed that higher levels of fibrinogen degradation products are associated 
with larger necrotic core. ${ }^{36}$ Moreover, D-dimer is also found to be related to the severity and complexity of atherosclerosis within the coronary tree and throughout the vessel system. ${ }^{37-39}$ Theoretically, sub-endothelial contents from these unstable lesions might directly activate the coagulation cascade, ${ }^{40-43}$ causing a continuous prothrombotic state, which therefore drives up D-dimer levels and the risk of future ischemia. ${ }^{44}{ }^{46}$ Besides, clinical studies also show that patients with elevated D-dimer acquire higher risk of no-reflow and heart failure after $\mathrm{PCI},{ }^{28,47}$ which could be results of massive thrombus within the coronary artery, microvascular embolism, and ongoing thrombosis within the circulation. In sum, elevations of D-dimer represented the impacts from the activation of the coagulation system. Assessments of D-dimer might have provided extra information regarding the prothrombotic tendency and the vulnerable lesions.

\section{Risk Stratifications Based on Age-Adjusted Elevation of D-Dimer}

As the established model was mainly based on pre-existing risk factors, hemodynamics, and angiographical details of culprit lesions, the addition of D-dimer might have subsidized the model by measuring the prognostic impacts regarding the vulnerable lesions, overall atherosclerotic burden, and systematic prothrombotic states. Physicians could therefore make use of age-adjusted elevation of D-dimer to back up the assessment for long-term risk of ischemia. ${ }^{12,13}$ For patients with elevated levels of D-dimer as adjusted by age, more intensive antithrombotic treatments should be considered to avoid future ischemic events. More potent P2Y12 inhibitors should be recommended, while low dose of oral anticoagulants could be considered for further risk reduction, which has been proved to be effective in reaching lower levels of D-dimer. ${ }^{48}$ Detailed intravascular imaging examinations (e.g., intravascular ultrasound, optical coherence tomography) would be helpful for assessment of lesion stability of non-culprit vessels and evaluation for the necessity of complete revascularizations. For patients with normal levels of D-dimer as adjusted by age, routine antiplatelet medications would be adequate for long-term management.

\section{Limitations}

The general limitations of this study are as follow. Firstly, patients in this study were retrospectively recruited. Although D-dimer was measured prospectively, residual confounding could still be present, and the causality between the elevation of D-dimer and outcomes could not be fully defined. In addition, blood samples for test of D-dimer were collected after PCI procedures, during which D-dimer levels could be affected by various invasive procedures and antithrombotic medications. Although age-adjusted D-dimer remained an independent predictor of adverse events in the current analysis, future studies are warranted to investigate whether D-dimer levels measured at admission could offer more accurate risk prediction. Besides, some examinations were not available due to limited resources, like imaging tests for infarct size, which should be adjusted in multivariable analysis to reduce the false effect in well-designed prospective studies. Moreover, this observational study was accomplished in a single institute. Although the sample size was large enough, the extrapolation of conclusions in the current study might require further validation.

\section{Conclusions}

High D-dimer levels, as defined by age-adjusted thresholds, was an independent predictor of adverse outcomes for ACS patients treated by PCI. When combined with clinical risk factors, age-adjusted elevation of D-dimer significantly improved the risk predictions for future mortality.

\section{Funding}

This study was supported by the National Natural Science Foundation of China (81970308), the Fund of "Sanming" Project of Medicine in Shenzhen (SZSM201911017), Shenzhen Key Medical Discipline Construction Fund (SZXK001), and the Chinese Academy of Medical Sciences Innovation Fund for Medical Sciences (2016-I2M-1-009).

\section{Disclosure}

The authors declare that they do not have any conflicts of interest. 


\section{References}

1. Righini M, Van Es J, Den Exter PL, et al. Age-adjusted D-dimer cutoff levels to rule out pulmonary embolism: the ADJUST-PE study. JAMA. 2014;311(11):1117-1124. doi:10.1001/jama.2014.2135

2. Tritschler T, Kraaijpoel N, Le Gal G, Wells PS. Venous thromboembolism: advances in diagnosis and treatment. JAMA. 2018;320(15):1583-1594. doi:10.1001/jama.2018.14346

3. Naruse H, Ishii J, Takahashi H, et al. Prognostic value of combination of plasma D-dimer concentration and estimated glomerular filtration rate in predicting long-term mortality of patients with stable coronary artery disease. Circ J. 2017;81(10):1506-1513. doi:10.1253/circj.CJ-16-1272

4. Simes J, Robledo KP, White HD, et al. D-dimer predicts long-term cause-specific mortality, cardiovascular events, and cancer in patients with stable coronary heart disease. Circulation. 2018;138(7):712-723. doi:10.1161/CIRCULATIONAHA.117.029901

5. Akgul O, Uyarel H, Pusuroglu H, et al. Predictive value of elevated D-dimer in patients undergoing primary angioplasty for ST elevation myocardial infarction. Blood Coagul Fibrinolysis. 2013;24(7):704-710. doi:10.1097/MBC.0b013e3283610396

6. Erkol A, Oduncu V, Turan B, et al. The value of plasma D-dimer level on admission in predicting no-reflow after primary percutaneous coronary intervention and long-term prognosis in patients with acute ST segment elevation myocardial infarction. $J$ Thromb Thrombolys. 2014;38 (3):339-347. doi:10.1007/s11239-013-1044-3

7. Kikkert WJ, Claessen BE, Stone GW, et al. D-dimer levels predict ischemic and hemorrhagic outcomes after acute myocardial infarction: a HORIZONS-AMI biomarker substudy. J Thromb Thrombolys. 2014;37(2):155-164. doi:10.1007/s11239-013-0953-5

8. Tello-Montoliu A, Marin F, Roldan V, et al. A multimarker risk stratification approach to non-ST elevation acute coronary syndrome: implications of troponin T, CRP, NT pro-BNP and fibrin D-dimer levels. J Intern Med. 2007;262(6):651-658. doi:10.1111/j.1365-2796.2007.01871.x

9. Sarli B, Akpek M, Baktir AO, et al. Impact of D-dimer level on postinterventional coronary flow and in-hospital MACE in ST-segment elevation myocardial infarction. Herz. 2015;40(3):507-513. doi:10.1007/s00059-013-4029-2

10. Mjelva OR, Ponitz V, Brugger-Andersen T, Grundt H, Staines H, Nilsen DW. Long-term prognostic utility of pentraxin 3 and D-dimer as compared to high-sensitivity C-reactive protein and B-type natriuretic peptide in suspected acute coronary syndrome. Eur J Prev Cardiol. 2016;23 (11):1130-1140. doi:10.1177/2047487315619733

11. Pineda J, Marin F, Marco P, et al. The prognostic value of biomarkers after a premature myocardial infarction. Int J Cardiol. 2010;143(3):249-254. doi:10.1016/j.ijcard.2009.02.019

12. Zhao X, Liu C, Zhou P, et al. Estimation of major adverse cardiovascular events in patients with myocardial infarction undergoing primary percutaneous coronary intervention: a risk prediction score model from a derivation and validation study. Front Cardiovasc Med. 2020;7:603621. doi: $10.3389 / \mathrm{fcvm} .2020 .603621$

13. Chen R, Liu C, Zhou P, et al. Prognostic value of D-dimer in patients with acute coronary syndrome treated by percutaneous coronary intervention: a retrospective cohort study. Thromb J. 2021;19(1):30. doi:10.1186/s12959-021-00281-y

14. Favaloro EJ, Franchini M, Lippi G. Aging hemostasis: changes to laboratory markers of hemostasis as we age - a narrative review. Semin Thromb Hemost. 2014;40(6):621-633. doi:10.1055/s-0034-1384631

15. Haase C, Joergensen M, Ellervik C, Joergensen MK, Bathum L. Age- and sex-dependent reference intervals for D-dimer: evidence for a marked increase by age. Thromb Res. 2013;132(6):676-680. doi:10.1016/j.thromres.2013.09.033

16. McBane RD 2nd, Hardison RM, Sobel BE. Comparison of plasminogen activator inhibitor-1, tissue type plasminogen activator antigen, fibrinogen, and D-dimer levels in various age decades in patients with type 2 diabetes mellitus and stable coronary artery disease (from the bari $2 \mathrm{D}$ trial). $\mathrm{Am}$ J Cardiol. 2010;105(1):17-24. doi:10.1016/j.amjcard.2009.08.643

17. van Es N, van der Hulle T, van Es J, et al. Wells rule and D-Dimer testing to rule out pulmonary embolism: a systematic review and individual-patient data meta-analysis. Ann Intern Med. 2016;165(4):253-261. doi:10.7326/M16-0031

18. Douma RA, le Gal G, Sohne M, et al. Potential of an age adjusted D-dimer cut-off value to improve the exclusion of pulmonary embolism in older patients: a retrospective analysis of three large cohorts. $B M J .2010 ; 340: \mathrm{c} 1475$. doi:10.1136/bmj.c1475

19. Ibanez B, James S, Agewall S, et al. 2017 ESC guidelines for the management of acute myocardial infarction in patients presenting with ST-segment elevation: the task force for the management of acute myocardial infarction in patients presenting with ST-segment elevation of the European Society of Cardiology (ESC). Eur Heart J. 2018;39(2):119-177. doi:10.1093/eurheartj/ehx393

20. Thygesen K, Alpert JS, Jaffe AS, et al. Fourth universal definition of myocardial infarction (2018). Eur Heart J. 2019;40(3):237-269.

21. Collet JP, Thiele H, Barbato E, et al. 2020 ESC Guidelines for the management of acute coronary syndromes in patients presenting without persistent ST-segment elevation. Eur Heart J. 2021;42(14):1289-1367. doi:10.1093/eurheartj/ehaa575

22. Newson RB. Comparing the predictive powers of survival models using Harrell's C or Somers' D. Stata J. 2010;10(3):339-358. doi:10.1177/ $1536867 X 1001000303$

23. Pencina MJ, D’Agostino RB Sr, Steyerberg EW. Extensions of net reclassification improvement calculations to measure usefulness of new biomarkers. Stat Med. 2011;30(1):11-21. doi:10.1002/sim.4085

24. Lunt M. Mark Lunt's personal home page. Available from: http://personalpagesmanchesteracuk/staff/marklunt/. Accessed January 20 , 2020.

25. Teunissen PFA, Tijssen R, van Montfoort ML, et al. Kinetics of coagulation in ST-elevation myocardial infarction following successful primary percutaneous coronary intervention. Thromb Res. 2016;137:64-71. doi:10.1016/j.thromres.2015.11.017

26. Kyrle PA, Eichinger S. D-dimer for long-term risk prediction in patients after acute coronary syndrome. Circulation. 2018;138(7):724-726. doi:10.1161/CIRCULATIONAHA.118.033670

27. Giannitsis E, Mair J, Christersson C, et al. How to use D-dimer in acute cardiovascular care. Eur Heart J Acute Cardiovasc Care. 2017;6(1):69-80. doi:10.1177/2048872615610870

28. Zhang X, Wang S, Liu J, et al. D-dimer and the incidence of heart failure and mortality after acute myocardial infarction. Heart. 2020;107 (3):44-237.

29. Malik AH, Yandrapalli S, Shetty SS, Aronow WS, Cooper HA, Panza JA. Meta-analysis of dual antiplatelet therapy versus monotherapy with P2Y12 inhibitors in patients after percutaneous coronary intervention. Am J Cardiol. 2020;127:25-29. doi:10.1016/j.amjcard.2020.04.027

30. Mavrakanas TA, Chatzizisis YS, Gariani K, et al. Duration of dual antiplatelet therapy in patients with CKD and drug-eluting stents: a meta-analysis. Clin J Am Soc Nephrol. 2019;14(6):810-822. doi:10.2215/CJN.12901018 
31. Wu Y, Song Y, Pan Y, Gong Y, Zhou Y. Long-term and short-term duration of thienopyridine therapy after coronary stenting in patients with chronic kidney disease a meta-analysis of literature studies. Platelets. 2020;31(4):483-489. doi:10.1080/09537104.2019.1647528

32. Yu T, Jiao Y, Song J, et al. Hospital mortality in acute coronary syndrome: adjustment of GRACE score by D-dimer enables a more accurate prediction in a prospective cohort study. BMC. 2019;19(1):252. doi:10.1186/s12872-019-1239-4

33. Pencina MJ, D'Agostino RB, Pencina KM, Janssens AC, Greenland P. Interpreting incremental value of markers added to risk prediction models. Am J Epidemiol. 2012;176(6):473-481. doi:10.1093/aje/kws207

34. Cook NR. Use and misuse of the receiver operating characteristic curve in risk prediction. Circulation. 2007;115(7):928-935. doi:10.1161/ CIRCULATIONAHA.106.672402

35. Kothari H, Nguyen AT, Yang X, et al. Association of D-dimer with plaque characteristics and plasma biomarkers of oxidation-specific epitopes in stable subjects with coronary artery disease. J Cardiovasc Transl Res. 2018;11(3):221-229. doi:10.1007/s12265-018-9790-4

36. Corban MT, Hung OY, Mekonnen G, et al. Elevated levels of serum fibrin and fibrinogen degradation products are independent predictors of larger coronary plaques and greater plaque necrotic core. Circ J. 2016;80(4):931-937. doi:10.1253/circj.CJ-15-0768

37. Kumakura H, Fujita K, Kanai H, et al. High-sensitivity c-reactive protein, lipoprotein (a) and homocysteine are risk factors for coronary artery disease in Japanese patients with peripheral arterial disease. $J$ Atheroscler Thromb. 2015;22(4):344-354. doi:10.5551/jat.25478

38. de Maat MP, Bladbjerg EM, Drivsholm T, Borch-Johnsen K, Møller L, Jespersen J. Inflammation, thrombosis and atherosclerosis: results of the Glostrup study. J Thromb Haemost. 2003;1(5):950-957. doi:10.1046/j.1538-7836.2003.00213.x

39. Zhang L, Hailati J, Ma X, et al. Analysis of risk factors for different subtypes of acute coronary syndrome. J Int Med Res. 2021;49 (5):3000605211008326.

40. Frangogiannis NG. Pathophysiology of myocardial infarction. Compr Physiol. 2015;5(4):1841-1875.

41. Connolly SJ, Eikelboom JW, Bosch J, et al. Rivaroxaban with or without aspirin in patients with stable coronary artery disease: an international, randomised, double-blind, placebo-controlled trial. Lancet. 2018;391(10117):205-218. doi:10.1016/S0140-6736(17)32458-3

42. Eikelboom J. Clopidogrel does not suppress blood markers of coagulation activation in aspirin-treated patients with non-ST-elevation acute coronary syndromes. Eur Heart J. 2002;23(22):1771-1779. doi:10.1053/euhj.2000.3234

43. Mahé I, Drouet L, Chassany O, et al. D-dimer: a characteristic of the coagulation state of each patient with chronic atrial fibrillation. Thromb Res. 2002;107(1-2):1-6. doi:10.1016/S0049-3848(02)00184-6

44. Tomaniak M, Katagiri Y, Modolo R, et al. Vulnerable plaques and patients: state-of-the-art. Eur Heart J. 2020;41(31):2997-3004. doi:10.1093/ eurheartj/ehaa227

45. Stefanadis C, Antoniou CK, Tsiachris D, Pietri P. Coronary atherosclerotic vulnerable plaque: current perspectives. J Am Heart Assoc. 2017;6(3). doi:10.1161/JAHA.117.005543

46. Prisco D, Antonucci E, Fedi S, et al. D-dimer increase after percutaneous transluminal angioplasty and clinical recurrence after primary revascularization in acute myocardial infarction? A pilot study. Clin Exp Med. 2001;1(4):219-224. doi:10.1007/s102380100006

47. Gong X, Lei X, Huang Z, et al. D-dimer level predicts angiographic no-reflow phenomenon after percutaneous coronary intervention within 2-7 days of symptom onset in patients with ST-segment elevation myocardial infarction. J Cardiovasc Transl Res. 2020;14(4):34-728.

48. AlKhalfan F, Kerneis M, Nafee T, et al. D-dimer levels and effect of rivaroxaban on those levels and outcomes in patients with acute coronary syndrome (an ATLAS ACS-TIMI 46 trial substudy). Am J Cardiol. 2018;122(9):1459-1464. doi:10.1016/j.amjcard.2018.07.032

Clinical Interventions in Aging

Dovepress

\section{Publish your work in this journal}

Clinical Interventions in Aging is an international, peer-reviewed journal focusing on evidence-based reports on the value or lack thereof of treatments intended to prevent or delay the onset of maladaptive correlates of aging in human beings. This journal is indexed on PubMed Central, MedLine, CAS, Scopus and the Elsevier Bibliographic databases. The manuscript management system is completely online and includes a very quick and fair peer-review system, which is all easy to use. Visit http://www.dovepress.com/testimonials.php to read real quotes from published authors.

Submit your manuscript here: https://www.dovepress.com/clinical-interventions-in-aging-journal 